case meteorological assumptions do the calculations show that the dose would exceed $100 \mathrm{mSv}$ in a small sector (an area less than 1 square kilometre).

\section{Amounts of activated material}

During its life a fusion power plant would generate, from component replacement and decommissioning, radioactive material similar in volume to that from a fission plant, but of considerably lower radiotoxicity. After 10 to 100 years, integral fusion radiotoxicity indices are comparable (ie 4 to 5 orders of magnitude below fission plants) to those for ashes (containing small quantities of uranium, thorium and their derivatives) from coal-fired plants, having delivered the same electrical energy over their lifetimes. The total activated masses are around 65000 tonnes. The assessments take into account 'commercial impurities' and confirm that the dominant portion of the radioactivity stems from the plasma facing components.

\section{Waste management and disposal}

The IAEA has recommended activity limits by nuclide for the release of activated materials from regulatory control by a process called 'clearance'. On this basis the most recent studies for the three models yield the following overall picture: 35 to $45 \%$ of the reactor mass could be cleared, 50 to $65 \%$ could be recycled (either by hand or with remote handing) and only a few per cent may need repository disposal.

In conclusion, fusion has a significant potential for safety. There is no uncontrolled power runaway. In the case of a total loss of active cooling the low decay heat strongly limits the mobilisation of radioactivity and excludes gross melting of reactor structures. The present view is that there would be no rupture of the radioactivity confinement due to internal events or external events with occurrence rates larger than $10^{-7}$ per annum and that these events can be covered by the design. Evacuation is not considered to be a mat- ter of real concern.

Over their lifetimes fusion power plants would generate activated material similar in volume to that of fission reactors but different in that the long-term radiotoxicity would be very much lower. An important issue is the use of advanced low activation materials, and of clearance and recycling which would further ease the management of radioactive waste. Only a small fraction of the activated material may need repository disposal.

The assessments confirm that safety and environmental advantages of fusion are not entirely inherent to the fusion process but rest also on the choice of materials and on design decisions.

Overall, the studies place on a firmer footing the judgement that fusion provides ultimate safety margins thanks to the self-limiting nature of the basic reaction, modest radiotoxicity of the inventories, passive means of decay heat removal and moderate confinement requirements.

\title{
Fusion Reactor Materials
}

\author{
Gilles Le Marois \\ CEA Grenoble, France
}

The selection of appropriate materials for fusion reactors relies on a trade-off between multiple requirements which are mainly driven by economic, safety and environmental factors. The selection is particularly important for the most highly exposed parts of the machine such as the blanket structure. The following issues must be considered in this respect: - The components need to be suitable for operating under severe thermomechanical loadings and intense irradiation by energetic neutrons up to high dose rate: fluence of up to 200 displacements per atom (dpa) are expected for these plasma-facing materials.

- Public acceptance of fusion is tied to its pledge of cleaner energy. Therefore low activation materials allowing firstly a significant reduction in the risk of exposure of personnel to radiation damage in case of an accident and secondly solving the problem of long life wastes, are an attractive solution for these components in a commercial reactor.

- Making fusion economically viable requires high energy conversion efficiency and low maintenance. Materials able to work at elevated temperature with high reliability will thus need to be developed. - In addition, highly advanced manufacturing processes will be developed to cope with other constraints such as complex shapes and low leak level requirements.

A number of classes of materials able to fulfill these requirements have been identified from a reasonable extrapolation of current knowledge. The choice of a blanket structural material has also to be combined with those of the coolant, of other blanket materials and to be discussed on the basis of a global definition of the machine, taking into account their technological limits, considering their specific function and the necessity to reduce their maintenance.

\section{Martensitic steels}

Due to their excellent resistance to irradiation induced swelling, low thermal expansion and high thermal conductivity allied to industrial maturity, ferritic/ martensitic (F/M) steels have been selected in the EU and Japan as the reference blanket structural material for the DEMO reactor. Recent irradiation testing in a fast breeder reactor up to 160 dpa has shown promising results and one can contemplate using these alloys for the internal structure of a fusion reactor.

The embrittlement induced by low temperature irradiation has been considered as a key issue for using the $\mathrm{F} / \mathrm{M}$ steels in a fusion reactor. Indeed F/M steels are ductile at high temperature but brittle at low temperature. The transition from ductile to brittle mode of failure occurs at a temperature called DBTT. The DBTT increases with irradiation level and the steel can become brittle at reactor working temperatures. Therefore efforts are being made to shift down this critical temperature by improving the steel chemical composition but without introducing elements that would form longlived isotopes or unacceptable corrosion behaviour. The present information on steel irradiation testing indicates that 9CrWTaV steel exhibits the best compromise between low irradiation hardening, low activation and resistance to corrosion. Control of the impurities that have the strongest impact on the long term radioactive activity is a major goal in demonstrating the feasibility of producing a low activation material on an industrial scale. This work is now in progress.

At high temperature, up to $550^{\circ} \mathrm{C}$, irradiation hardening is lower or does not occur. In contrast, ductility can be lower compared to the unirradiated state. This is usually due to segregation of $\mathrm{He}$ at the grain boundaries. Swelling could appear. 
F/M steels are mainly preferred to austenitic steels as they are less sensitive to this phenomenon. The main uncertainties come from the higher $\mathrm{He} / \mathrm{dpa}$ ratio resulting from the fusion neutron spectra that could cause the DBTT to shift further up.

Above $550^{\circ} \mathrm{C}$, the strength of conventional F/M steels rapidly declines. For this kind of material, $550^{\circ} \mathrm{C}$ is therefore considered to be the upper service temperature. Using F/M steels reinforced by a dispersion of strengthening oxide, this upper temperature could be increased by $100^{\circ} \mathrm{C}$.

Another possible drawback are the ferro-magnetic properties of these alloys and their interaction with the magnetic field has to be precisely assessed.

Implementation and joining of $\mathrm{F} / \mathrm{M}$ steels are an important goal due to the complex geometries of the blanket modules and the necessity of reducing the leak level. For that purpose, advanced techniques based on solid or powder hot isostatic pressing (HIP) are under development.

\section{Non ferrous alloys and ceramics}

For higher working temperatures and improved radiological behaviour on the short and medium terms, non-ferrous alloys as vanadium, titanium or chromium alloys and the $\mathrm{SiC} / \mathrm{SiC}$ ceramic composite are being considered. These types of materials will, however, necessitate several tens of years of R\&D before being suitable for nuclear components.

\section{Vanadium alloys}

Most of the R\&D is carried out in the US. Due to their high sensitivity to oxygen, they can only be associated with liquid lithium as a coolant. Brittleness of these alloys is caused by irradiation at low temperature and the presence of other gaseous impurities such as nitrogen and hydrogen.

\section{$\mathrm{SiC} / \mathrm{SiC}$ composites}

Mainly studied in the EU and Japan, they potentially offer the best compromise in terms of performance (they can be operated up to at least $1100^{\circ} \mathrm{C}$ ), maintenance and waste. Their possible use remains subject to an improvement of their physical properties (thermal and electrical conductivities), of their irradiation sensitivity and to a reduction in their cost.

\section{Titanium and chromium alloys}

Considered primarly for their low neutron activation, their use remains subject to improvements. $\mathrm{Cr}$ alloys and beta $\mathrm{Ti}$ alloys are extremely brittle after irradiation. Embrittlement of the alpha Ti alloys is also caused by hydride formation. Progress has been made to overcome these drawbacks. For $\mathrm{Cr}$, a powder route aiming at a finer microstructure is being studied inj order to improve its ductility. The coating of Ti to protect it against $\mathrm{H}$ and the introduction of carbon fibers to reinforce the alloy are under evaluation.

A comparison between these different alloys is presented in the table below.

\section{Irradiation testing}

Present nuclear devices are not well adapted for irradiation testing under relevant fusion conditions, with a representative neutron spectra. A progressive approach has been proposed in the EU programme to take into account limited available resources. It includes the 3 following steps: - Modelling of the irradiation effects using small devices and codes to investigate effects of alloying and stabilizing elements, impurities, and so on.

- Material and small components testing in a device such as IFMIF (for which no decision about place and construction has been made). The IFMIF D+- $\mathrm{Li}$ accelerator is a high flux, high energy neutron source that allows irradiation of acceptably large volumes: $1 \mathrm{dpa} /$ year on $50 \mathrm{dm}^{3}$ and 50 dpa/year on $0.1 \mathrm{dm}^{3}$ can be achieved. A detailed design of the device has been completed and it could be operational by 2005 if construction takes place.

- At a later stage, component and scale effects testing in realistic conditions in a bigger volumetric source device, the socalled VNS.

In conclusion, $R \& D$ activities on materials for fusion reactors in the EU aim at the development of the following: promising F/M steels to be used on the short term, advanced materials such as $\mathrm{SiC} / \mathrm{SiC}$ for reactor performance improvement and specific tools, processes for materials implementation and characterization.

Considering the complexity of a fusion device, these developments have to be carried out in the frame of reactor studies where the possible choices (including plasma models, size of the machine, safety, cost, impact on the environment, technological limits imposed by the materials and their implementation) will be discussed globally and compared.

\section{Structural materials for fusion reactor blankets}

$\checkmark$ good $x$ bad $\checkmark \checkmark$ very good $x x$ very bad etc

Low activation
short term (safety)
long term (wastes)
Irradiation behaviour
swelling
embrittlement
$\begin{aligned} & \text { Working temperature } \\ & \text { (energy efficiency) }\end{aligned}$
$\begin{aligned} & \text { Compatibility } \\ & \text { with coolant } \\ & \text { with } \mathrm{H} \\ & \text { with magnetic field }\end{aligned}$

V alloys (V4Ti4Cr)
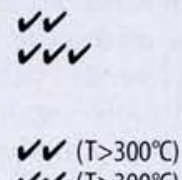

$\checkmark v\left(T>300^{\circ} \mathrm{C}\right)$

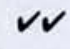

$x^{(L i)}$
$x$

Can only be combined with liquid lithium as a coolant, insulating coating is required (due to MHD effect), reduction of $\mathrm{Nb}$ content (an impurity)

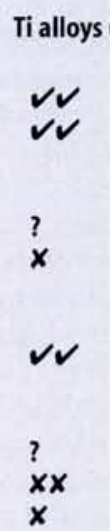

Optimization of the $\beta$ alloy microstructure to reduce its brittleness, reduction of $\mathrm{Al}$ content

\section{CR alloys $(\mathrm{Cr})$}

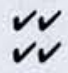

$?$

$x x$

$?$

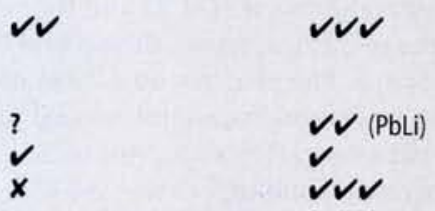

Optimization of the microstructure to reduce its brittleness

A new material, optimization of fibres and impregnation process to improve irradiation behaviour 